\title{
A Multi-User Cooperative Diversity for Wireless Local Area Networks
}

\author{
Jun CHEN ${ }^{1}$, Karim DJOUANI ${ }^{2}$ \\ ${ }^{I}$ LISSI Lab., University Paris 12 and CEDRIC-CNAM Paris, France \\ ${ }^{2}$ Member, IEEE, F'SATIE/TUT Pretoria, South Africa and LISSI Lab., University Paris 12, France \\ E-mail: ${ }^{1}$ chen_ju@auditeur.cnam.fr, ${ }^{2}$ djouani@univ-paris12.fr \\ Received on November 12, 2007; revised and accepted on May 3, 2008
}

\begin{abstract}
In this paper, an idea of using space-time block coding (STBC) in multi-user cooperative diversity has been exploited to improve the performance of the transmission in wireless local area networks. The theoretical and simulation results show that, using STBC approaches can always achieve the better performance than existing techniques without introducing the space-time coding. By analyzing the throughput and frame error ratio (FER) of the two different STBC cooperative schemes, we find the trade-off between throughput and reliability. The location of the relay is crucial to the performance, which supposes a rule for future crosslayer design.
\end{abstract}

Keywords: Multiple-input-multiple-output (MIMO), Cooperation, Space-time Block Coding (STBC).

\section{Introduction}

Diversity is a powerful technique to mitigate fading and improve robustness to interference [1], which refers to the method by conveying the signal to the receiver over multiple independently signal fading channels. The conventional view of transmit diversity is that a single wireless terminal transmits using an array of multipleantennas so that the paths from each antenna to the destination with independently fading. The recent research work in this area is the space-time coding (STC) techniques that have been developed for multi-antenna arrays. STC is an effective coding technique that uses transmit diversity to combat the detrimental effects in wireless fading channels [7]. Unfortunately, transmit diversity methods based on multiple-input-multipleoutput (MIMO) approach are not applicable to many wireless systems because of the size, complexity, power or other constraints, as for instance, ad-hoc networks and sensor networks. On account of these reasons, cooperation between wireless terminals has been recently proposed as a means to provide transmit diversity as which shown in Figure 1, where S, R and D represent source, relay and destination terminal, respectively. A new method introduced in [2] and [3] to realize space diversity gain has been studied under the name of cooperative diversity. Traditional cooperative diversity transmits the same signals through two different channels as Figure 2. In the first time slot, the source communicates to the relay and to the destination at the same time; in the second time slot, just the relay retransmits the signal received at the first time-slot to the destination. The relay may simply forward the signal received from the source terminal or retransmit the estimates of the received symbols, obtained by detection. We call it as repeat cooperation. In this paper, we present a paradigm for cooperative diversity, which we term space-time block coding (STBC) cooperation [21], integrating user cooperation with STBC.

We summarize here the relevant contributions in the area of the cooperative diversity. Relay channels and spacetime code form the basis for our study. The classical threeterminal communication channels originally examined by van der Meulen [5]. For the channels with multiple information sources, Kramer and van Wijngaarden [6] consider a multiple access channel in which the sources communicate to one destination and share one relay.

Laneman et al. examines the mode of user cooperation diversity [2,3] and analyzes space time coding cooperative diversity in nonergodic settings using outage probability as a performance measure [4]. They 


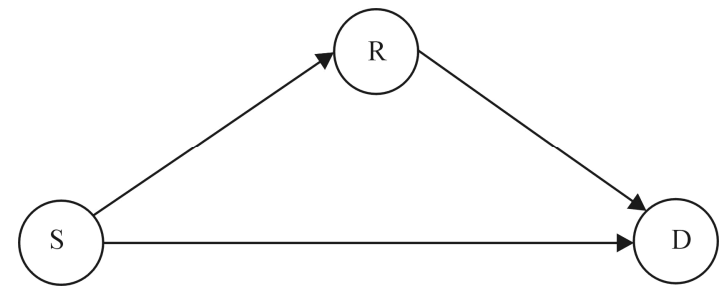

Figure 1. Single-relay cooperative diversity model.

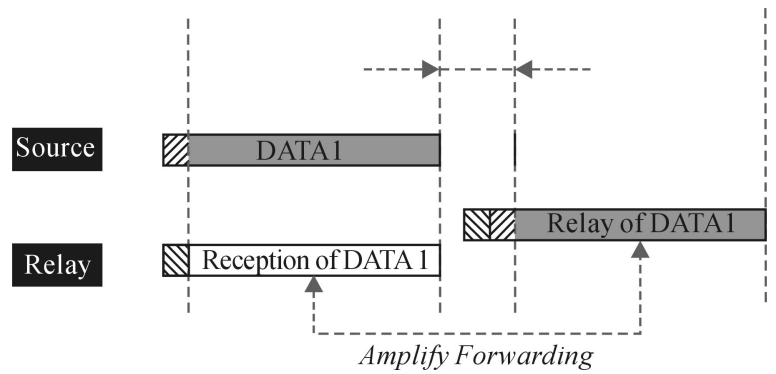

Figure 2. Time sequence of 2 time slots repeat cooperative diversity.

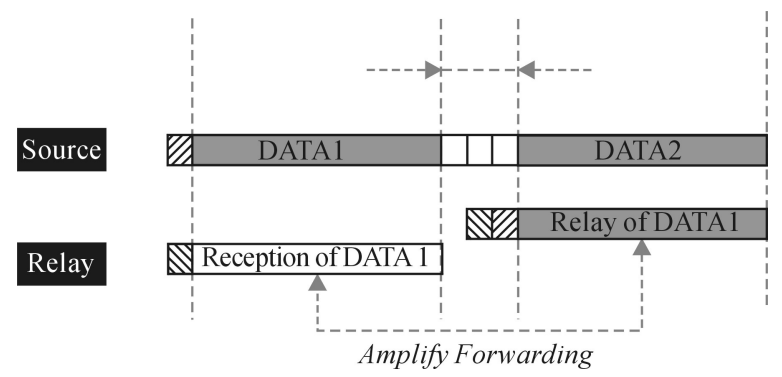

Figure 3. Time sequence of 2 time slots multi-user cooperative diversity.

demonstrated the extent to which space-time coding cooperative diversity achieves higher diversity order than repetition-based schemes for larger spectral efficiencies in theorem. The model they analyzed is a selective orthogonal amplify and forward (OAF) protocol, where source transmits the vector of encoded data in the first time slot and relay retransmits the received vector by adjusting the power. The non-orthogonal amplify-andforward (NAF) scheme was proposed by Nabar et al. $[8,9]$ for the single-relay channel, where source transmits all the time but the relay only transmits on even time slots. They consider three different time-division multiple-access-based cooperative protocols that vary the degree of broadcasting and receive collision in either the amplify-and-forward (AF) or decode-and-forward (DF) modes. And the results indicate that optimal space-time code design in the single relay case consists of satisfying the classical rank and determinant criteria for co-located antennas. These academic works sustain the possibility, existence and benefits for deploying space-time coding cooperative diversity protocols in practice.

This paper examines a new $2 \times 2$ full-rate space-time code (Golden-Code) [12] in the single-relay cooperative
NAF model. For source transmits in both two time slots, this protocol can achieve a higher throughput than that of the OAF protocol. And we here consider these two types of cooperative protocols and compare the performance between Golden-Code and the classical Alamouti code [10]. Besides the distinct benefits of the space-time code, we can see the trade-off between throughput and reliability during the transmission by analyzing the results of throughput and frame error rate. At the last part, we give a basic idea about the selection of relay.

Organization of the paper. This paper continues as follows: Section 2 outlines the multi-user cooperative diversity model. Section 3 explains STBC cooperative diversity. Section 4 shows the performance analysis by the simulation results. Section 5 summarizes our conclusions.

\section{Multi-User Cooperative Diversity Model}

We consider wireless network in which two terminals are communicating with a base station. The channel between each terminal and the base station are independent of each other, and independent of the channel between the terminals. All channels are subject to flat (frequency nonselective) fading in order to isolate the benefits of spatial diversity. Considering the multi-user cooperative diversity model, signal is to be transmitted from the source terminal $S$ to the destination terminal $D$ with the assistance of the relay terminal $R$. All the terminals are equipped with single antenna. Throughout the paper we assume that a terminal cannot transmit and receive simultaneously. the channels $S \rightarrow D, S \rightarrow R$ and $R \rightarrow D$ are known to the destination terminal.

The signal transmits procession is like following: During the first time slot, the source communicates with the relay and destination. In the second time slot, both the relay and source communicate with the destination. Figure 3 shows the detail of the time sequence.

In the AF relaying method [1], the relay simply amplifies and retransmits the signal received from the source (the signal received at the relay is distorted by fading and additive noise). No demodulation or decoding of the received signal is performed at relay in this case.

The signals received by the destination and relay in the first time slot can be defined as

and

$$
y_{s d}=w_{s d} h_{s d} x_{1}+n_{s d}
$$

$$
y_{s r}=w_{s r} h_{s r} x_{1}+n_{s r}
$$

respectively, where $w_{s d}^{2}$ and $w_{s r}^{2}$ are the average signal energies received by destination over channel $S \rightarrow D$ and $S \rightarrow R$, respectively [9]. $h_{s d}$ and $h_{s r}$ are the random, complex-valued and unit-power channel gains between $S$ $\rightarrow D$ and $S \rightarrow R . n_{s d} \backsim C N\left(O, N_{s d}, n_{s r} \sim C N(0, N s r)\right.$ is the 
additive noises, and in general $w_{s d}^{2} \neq w_{s r}^{2}$.

The energy of received signal (3) is given by

$$
E\left(\left|y_{s r}\right|^{2}\right)=E\left(\left|w_{s r} h_{s r} x_{1}\right|^{2}\right)+E\left(\left|n_{s r}\right|^{2}\right)=w_{s r}^{2} h_{s r}^{2}+N_{s r}
$$

In order to retransmit the signal with the same power as the sender did, the gain $\beta$ for the amplification is

$$
\beta=\sqrt{\frac{1}{w_{s r}^{2} h_{s r}^{2}+N_{s r}}}
$$

Then, the destination receives a superposition of relay and source during the second time slot:

$$
y_{2}=w_{s d} h_{s d} x_{2}+v_{r d} h_{r d} \beta y_{s r}+n_{r d}
$$

where $v_{r d}^{2}$ is the average signal energy received at the destination through channel $R \rightarrow D$, the definition of $h_{r d}$ and $n_{r d}$ are the similar to $h_{s r}$ and $n_{s r}$.

So the equation (5) can be rewritten as:

$$
y_{2}=w_{s d} h_{s d} x_{2}+v_{r d} w_{s r} \beta h_{r d} x_{1}+\tilde{n}
$$

where $\tilde{n} \backsim C N\left(0, N_{0}\right)$ with $N_{0}=v_{r d}^{2} h_{r d}^{2} \beta^{2} N_{s r}+N_{r d}$

As the summary, the transmission function of this cooperative diversity is

$$
\mathrm{y}=\mathrm{Hx}+\mathrm{n}
$$

where

$$
y=\left[\begin{array}{l}
y_{1} \\
y_{2}
\end{array}\right], x=\left[\begin{array}{l}
x_{1} \\
x_{2}
\end{array}\right]
$$

is the received signal vector and transmitted signal vector, respectively;

$$
n=\left[\begin{array}{c}
n_{s d} \\
v_{r d} h_{r d} \beta n_{s r}+n_{r d}
\end{array}\right]
$$

is the noise vector; and $\mathrm{H}$ is the $2 \times 2$ channel matrix given by

$$
H=\left[\begin{array}{cc}
w_{s d} & 0 \\
w_{s r} v_{r d} \beta h_{s r} h_{r d} & w_{s d} h_{s d}
\end{array}\right]
$$

Assuming that the channel coefficient matrix $\mathrm{H}$ is known or can be estimated, Maximum Likelihood (ML) decoding can be used at receiver to fully explore the diversity advantage of the scheme. In equation (9), the noise of first time slot and second time slot do not have the same powers, the ML estimation can not be used directly. One solution is normalizing the received noise by a parameter $\rho$ as follows:

$$
\left[\begin{array}{c}
y_{1} \\
\rho y_{2}
\end{array}\right]=\left[\begin{array}{cc}
w_{s d} h_{s d} & 0 \\
\rho w_{s r} v_{r d} \beta h_{s r} h_{r d} & \rho w_{s d} h_{s d}
\end{array}\right]\left[\begin{array}{l}
x_{1} \\
x_{2}
\end{array}\right]+\left[\begin{array}{c}
n_{s d} \\
\rho \tilde{n}
\end{array}\right]
$$

where

$$
\rho=\sqrt{\frac{N_{r d}}{\beta^{2} v_{r d} h_{r d}^{2} N_{s r}+N_{r d}}}
$$

Then, equation (10) can be noted as $\tilde{y}=\tilde{H} \mathrm{x}+\tilde{n}$.
Assuming that the channel coefficient matrix $\tilde{H}$ is known or can be estimated, the ML estimate of the transmitted packets is presented as follows:

$$
\hat{x}=\arg \min _{x}\|\tilde{n}-\tilde{H} x\|_{F}^{2}
$$

where $\|\cdot\| F$ represents the Frobenius-2 norm, and $\mathrm{x}$ takes all possible finite values depending on the signal constellation.

\section{STBC Cooperation Model}

STC is a method employed to improve the reliability of data transmission in wireless systems by using multiple transmit antennas. It relies on redundant copies of a signal to the receiver in the hope that at least some of them may survive the physical path between transmission and reception. Space time codes may be split into two main types: Space-time trellis coding (STTC) [16] and STBC [17]. We are only concerned here with STBC which acts on a block of data at once (similarly to block coding) and provide only diversity gain, but are much less complex in implementation terms than STTC. Alamouti coding [10] and Golden-Code [12] are typical examples of STBC.

\subsection{Repeat Cooperation}

Firstly, we present the model shown in Figure 2, repeat cooperation transmits the same signals through two different channels. In the first time slot, the source communicates to the relay and to the destination at the same time; in the second time slot, just the relay retransmits the signal received at the first time slot to the destination. Then, the transmission function can be noted as follows:

$$
\begin{aligned}
& y_{1}=w_{s d} h_{s d} x_{1}+n_{s d} \\
& y_{2}=\beta v_{r d} w_{s r} h_{s r} h_{r d} x_{1}+\beta v_{r d} h_{r d} n_{s r}+n_{r d}
\end{aligned}
$$

The cooperative transmission function can be written as

$$
y=h x_{1}+n
$$

where

$$
\begin{aligned}
& y=\left[\begin{array}{l}
y_{1} \\
y_{2}
\end{array}\right], h=\left[\begin{array}{c}
w_{s d} h_{s d} \\
\beta v_{r d} w_{s r} h_{s r} h_{r d}
\end{array}\right] \\
& n=\left[\begin{array}{c}
n_{s d} \\
\beta v_{r d} h_{r d} n_{s r}+n_{r d}
\end{array}\right]
\end{aligned}
$$

\subsection{Alamouti Coding Cooperation}

Alamouti proposed a simple MIMO scheme that achieves a full diversity gain [17] with a simple ML 
decoding algorithm. The transmit signals are modulated using an M-ary modulation scheme, then the encoder takes a block of two modulated signals $s 1$ and $s 2$ in each encoding operation and sends it to the transmit antennas according to the code matrix:

$$
C=\left[\begin{array}{ll}
x_{1} & x_{2} \\
x_{3} & x_{4}
\end{array}\right]=\left[\begin{array}{cc}
s_{1} & -s_{2}^{*} \\
s_{2} & s_{1}^{*}
\end{array}\right]
$$

where * denotes complex conjugate. In this code matrix, the first column represents the first time slot (transmission period) in a $2 \times 2$ MIMO system [11] and the second column represents the second time slot. The first row corresponds to the signals transmitted from the first antenna and the second row corresponds to the signals transmitted from the second one. This implies that the signals are transmitting both in space (across two antennas) and time (two transmission intervals), that is to say, space-time coding.

The traditional Alamouti coding is designed for a two-transmit antenna system. Assuming the cooperative method using one-relay AF channel, we define $d_{1}=(x 1$, $\left.x_{2}\right)$ and $d_{2}=\left(x_{3}, x_{4}\right)$. Thus, in the first time slot, the source sends $d_{1}$, the relay and destination receive the signal; in the second time slot, the source and relay send $d 2$ and $x_{r}$ to destination respectively. Then the Alamouti coding cooperative transmission function can be written as

$$
\mathrm{Y}=\mathrm{HX}+\mathrm{N}
$$

where

$$
X=\left[\begin{array}{ll}
x_{1} & x_{2} \\
x_{3} & x_{4}
\end{array}\right], Y=\left[\begin{array}{ll}
y_{1} & y_{2} \\
y_{3} & y_{4}
\end{array}\right]
$$

are the transmitted and received signal matrix, respectively; channel matrix $\mathrm{H}$ and noise $\mathrm{N}$ are given by

$$
\begin{aligned}
& H=\left[\begin{array}{cc}
w_{s d} h_{s d} & 0 \\
\beta v_{r d} w_{s d} h_{s r} h_{r d} & w_{s r} h_{s d}
\end{array}\right] \\
& N=\left[\begin{array}{cc}
n_{s d 1} & n_{s d 2} \\
\beta v_{r d} h_{r d} n_{s r 1}+n_{r d 1}+n_{s d 3} & \beta v_{r d} h_{r d} n_{s r 2}+n_{r d 2}+n_{s d 4}
\end{array}\right]
\end{aligned}
$$

\subsection{Golden-Code Cooperation}

The Golden-Code is a STBC for $2 \times 2$ MIMO system as Figure 5, the coding matrix for the model is:

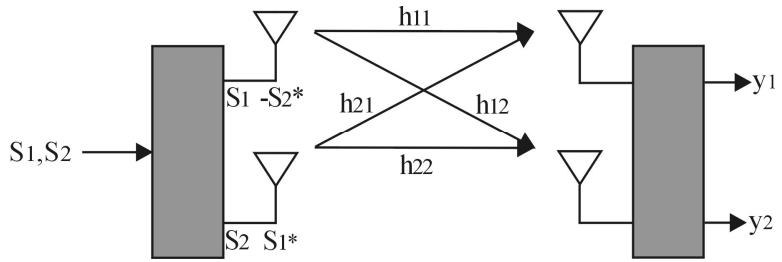

Figure 4. Alamouti coding in $2 \times 2$ MIMO model.

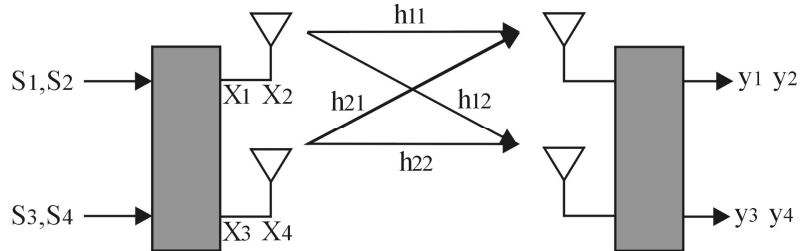

Figure 5. Golden-code in $2 \times 2$ MIMO model.

$$
C=\left[\begin{array}{ll}
x_{1} & x_{2} \\
x_{3} & x_{4}
\end{array}\right]=\frac{1}{\sqrt{5}}\left[\begin{array}{cc}
\alpha\left(s_{1}+\theta s_{2}\right) & \alpha\left(s_{3}+\theta s_{4}\right) \\
i \bar{\alpha}\left(s_{3}+\bar{\theta} s_{4}\right) & \bar{\alpha}\left(s_{1}+\bar{\theta} s_{2}\right)
\end{array}\right]
$$

where $s 1, s 2, s 3, s 4 \in Z[i]$ are the information signals, $\theta=\frac{1+\sqrt{5}}{2}, \bar{\theta}=\frac{1-\sqrt{5}}{2}, \alpha=1-i-i \theta, \bar{\alpha}=1-i-i \bar{\theta} \quad$ and the factor $\frac{1}{\sqrt{5}}$ is necessary for energy normalizing purposes [12].

The Golden-Code achieves the diversity multiplexing frontier [13], and in [12] the Golden-Code was proposed as a full rate and full diversity code for $2 \times 2$ MIMO systems.

To the cooperative method using one-relay AF channel, we define $d 1=\{(\mathrm{x} 1, \mathrm{x} 2)\}$ and $d 2=\{(\mathrm{x} 3, \mathrm{x} 4)\}$ which are transmitted in first time slot and second time slot, respectively. The transmission function is similar to equation (16).

\section{Numeral Results}

In this section, some simulations are presented to show the performances of the presented approaches. In the following simulations, Rayleigh model is used for the fading channel [20], each channel multi-path is a zero mean complex Gaussian random variable, and the distance between all the terminals is assumed to be same. Transmission energies follow the hypothesis as Table 1.

Table 1. Transmission energies in simulations.

\begin{tabular}{|c|c|c|c|}
\hline Protocol & \multicolumn{2}{|c|}{$1_{\text {st }}$ time slot } & $2_{\text {nd }}$ time slot \\
\hline \hline Cooperation & $w_{s d}^{2}=1.0$ & $w_{s r}^{2}=0.5$ & $v_{r d}^{2}=0.5$ \\
\hline MIMO & \multicolumn{2}{|c|}{$w_{11}^{2}=w_{12}^{2}=0.5$} & $v_{11}^{2}=v_{12}^{2}=0.5$ \\
\hline
\end{tabular}

The throughput was defined as the average number of available frames that were transmitted in a specific time slot. We performed a random experiment consisting of 10,000 repeated independent trials. The length of each frame was fixed to $N=600$ bits. Considering the multipack reception, the throughput can more than 1 .

\subsection{The Throughput Comparison between the Repeat Cooperation and STBC Cooperation}

We conducted comparisons between the STBC 
cooperation and repeat cooperation scheme. Figure 6 and Figure 7 show the results of throughput versus SNR, for $6 \mathrm{Mbps}$ and $12 \mathrm{Mbps}$ transmit rates, respectively. We observe that all the three schemes can achieve the maximum throughput with a high SNR $(>25 \mathrm{~dB})$. With a special coding method, Golden-Code cooperation scheme achieves a much higher throughput than the other two. Considering the coding matrix of Golden-Code, each row contains all the 4 original signals, which means the full-rate of the transmission. The cooperative method transmits 4 available signals $(\{\mathrm{s} 1 ; \mathrm{s} 2 ; \mathrm{s} 3 ; \mathrm{s} 4\})$ during 2 time slots, which means the maximum value of throughput is 2 .

As to amamouti coding scheme, each row of the coding matrix contains 2 original signals ( $s_{1}$ and $s_{2}$ ). In every time slot, the system transmits one signal and the conjugated signal of the other one, where $s_{1}^{*}$ and $s_{2}^{*}$ are surly the redundancy copies of the original signals. That is why only 2 available signals ( $s_{1}$ and $s_{2}$ ) can be obtained at the destination in this scheme while 4 available signals $\left(\left\{1 ; s_{2} ; s_{3} ; s_{4}\right\}\right)$ can be obtained by using Golden-Code scheme. Thus, by using two pair of conjugate signals, Alamouti coding scheme transmits 2 available signals during 2 time slots of the cooperative period, which means the maximum value of throughput can no more than 1 with the increasing of SNR.

Furthermore, as shown in Figure 2, repeat cooperation transmits one signal during the first time slot and retransmits the same one in the second. Clearly, repeat cooperation can just transmit 1 signal during the two time slots. Thus, its throughput is less than 0.5.

From the simulations, we see that with the help of STBC gains, the STBC cooperation is outperform repeat cooperation. And as a reasonable result of analysis and simulation, the Golden-Code cooperation can clearly achieve the best throughput among all the three schemes. This also proves that the design of the space-time code could impact the performance of the transmission.

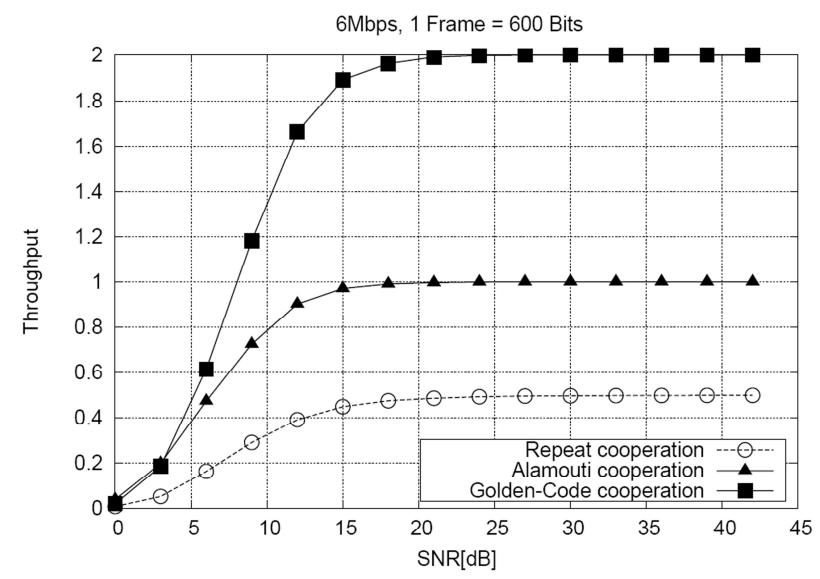

Figure 6. Throughput of STBC cooperation and repeat cooperation schemes (6Mbps).

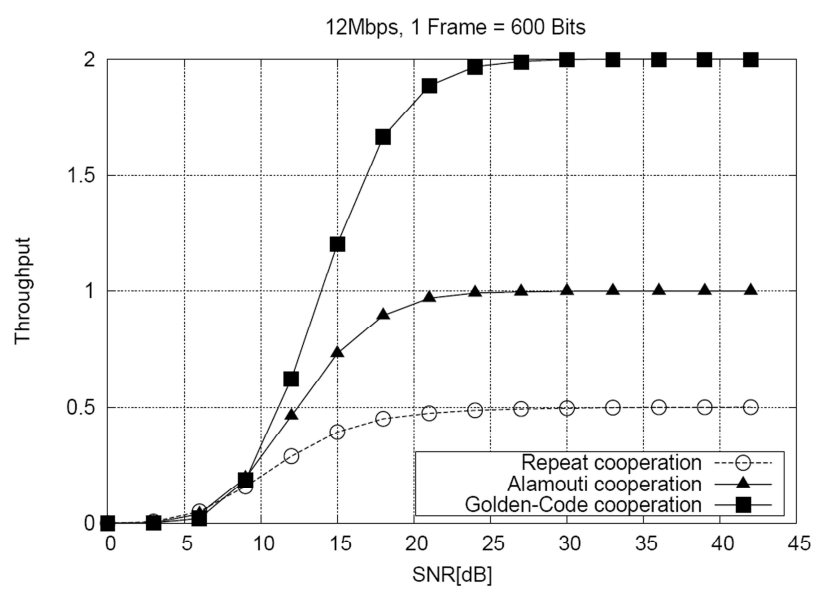

Figure 7. Throughput of STBC cooperation and repeat cooperation schemes (12Mbps).

\subsection{The FER Comparison between Non- ooperation, Repeat Cooperation and STBC Cooperation}

The simulation results of FER versus SNR between Noncooperation and cooperation schemes demonstrate again that the use of relay-assisted communication is not always beneficial when compared to direct transmission (Non-cooperation scheme) [8]. Figure 8 and Figure 9 reveal that the frame error rate of Non-cooperation communication is better than that of the simple repeat cooperation for a high SNR $(>35 \mathrm{~dB})$.

Further, as expected, cooperation with STBC is always preferred over Non-cooperation scheme. Thus from our simulations, we see that, performance using STBC cooperation improves significantly over Noncooperation demonstrating the advantage of using STBC cooperation. Between the two STBC cooperation schemes (Alamouti coding and Golden-Code), Alamouti coding method shows a better performance. As we discussed, Alamouti coding transmits the redundance

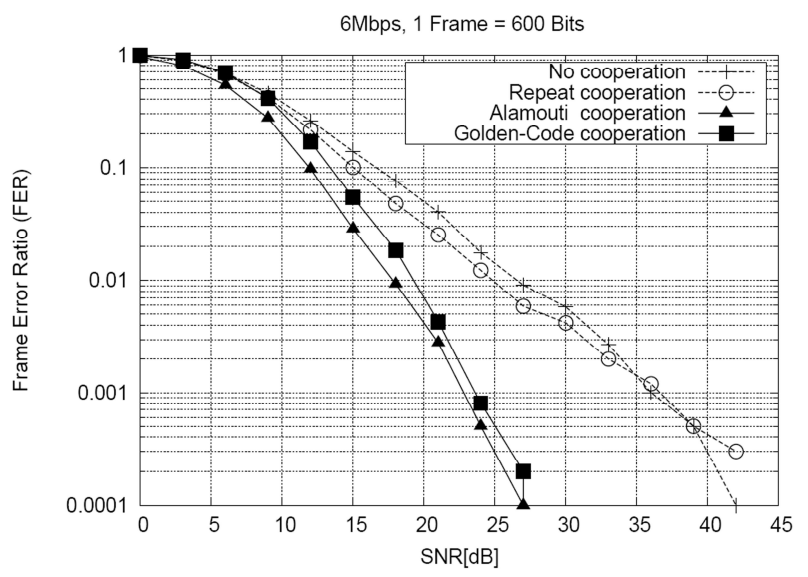

Figure 8. FER versus SNR for Non-cooperation and cooperation schemes (6Mbps). 


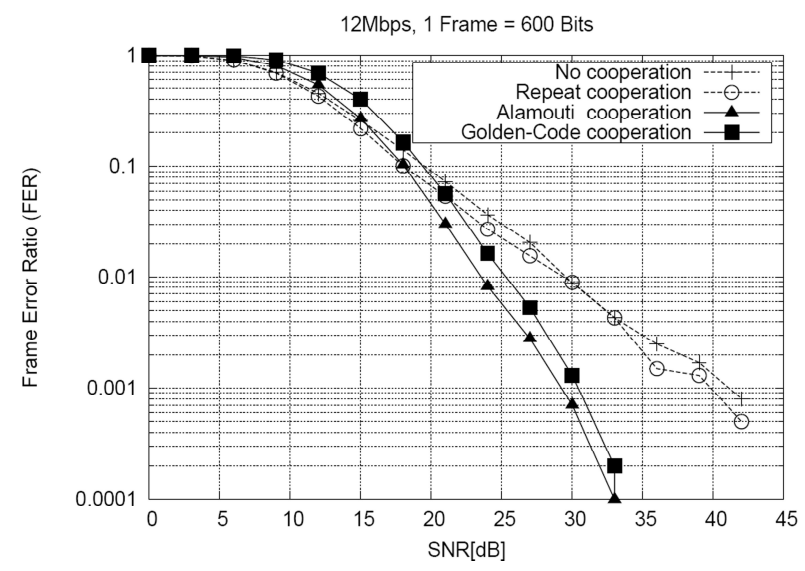

Figure 9. FER versus SNR for Non-cooperation and cooperation schemes (12Mbps).

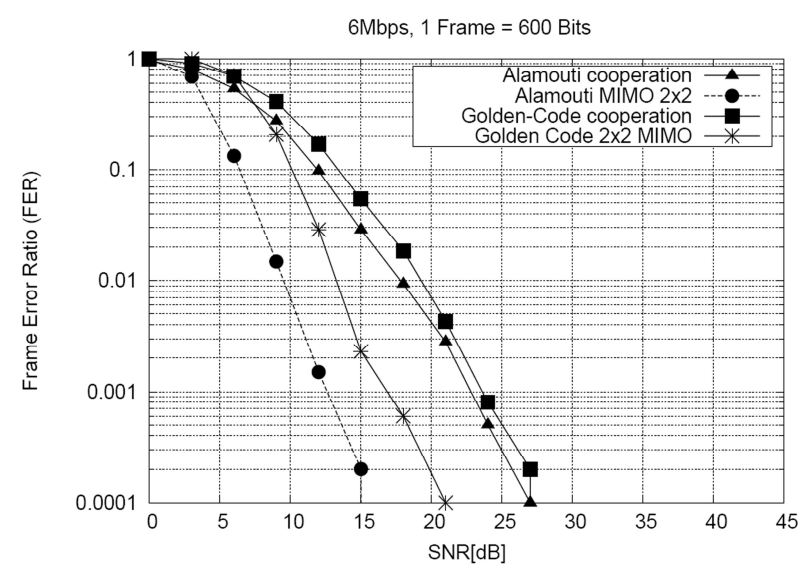

Figure 10. FER versus SNR for STBC in cooperation and MIMO schemes (6Mbps).

signals, a original and a conjugate. This is the reason that it has a lower error rate in the destination while GoldenCode just intersperses original signal among all parts of the transmit signals.

Comparing with the simulation results about the throughput of these two STBC cooperation schemes, we see that, Alamouti coding have a lower throughput but a higher reliability than that of Golden-Code. As a summary, there is always a trade-off between the throughput and the reliability.

\subsection{The FER Comparison between STB Cooperation and MIMO Schemes}

Figure 10 and Figure 11 show us the FER of cooperation and MIMO system referring to the different SNRs. According to the simulation results, the MIMO systems achieve lower FER than the corresponding cooperative schemes. This supports that MIMO channels allowing multiplexing gain $[14,15]$ which is absent in cooperative relaying channel since time is expended in the latter. Thus, using MIMO system always obtains the gain of spatial diversity. And as expected, the Alamouti coding method has a better performance than the corresponding
Golden-Code method. The simulation result demonstrates again that there is a trade-off between the throughput and the reliability.

\subsection{Effect via the Movements of the Relay}

The main building blocks of a wireless network design are rate control, power control, medium access (scheduling) and routing. These building blocks are divided in layers. Typically, routing is considered in a routing layer and medium access in a MAC-layer, whereas power control and rate control are sometimes considered in a PHY-layer and sometimes in a MAC-layer.

So far, the three stations $(S, R, D)$ were positioned equidistantly and therefore all the three channels had the fixed distance. Let us denote the distance between source and destination as $d_{s} d$; distance between source and relay as $d_{s r}$ and distance between relay and destination as $d r d$. Denote $S N R_{s d}, S N R s r$, SNRrd as SNR between the source and destination during the 2 time slots. We have

$$
\begin{aligned}
& S N R_{s r} \propto\left(\frac{1}{d_{s r}}\right)^{v} \\
& S N R_{r d} \propto\left(\frac{1}{d_{r d}}\right)^{v} \\
& S N R_{s d} \propto\left(\frac{1}{d_{s d}}\right)^{v}
\end{aligned}
$$

where $v$ is the path loss exponent. In the following analysis, we assume that $v=4$ for urban environment [18].

In this section, the relay is moved, so the distance between the relay and source, the relay and destination will change at the same time. The effects on the signal quality when moving the relay between the source and destination using Golden-Code cooperation with 6Mbps and $12 \mathrm{Mbps}$ transmission rate are shown in Figure 12 and Figure 13, respectively. In the simulations, the distance between the sender and the destination is set to one, and therefore the SNRs shown in the X-axis is only valid for the direct link $S \rightarrow D$.

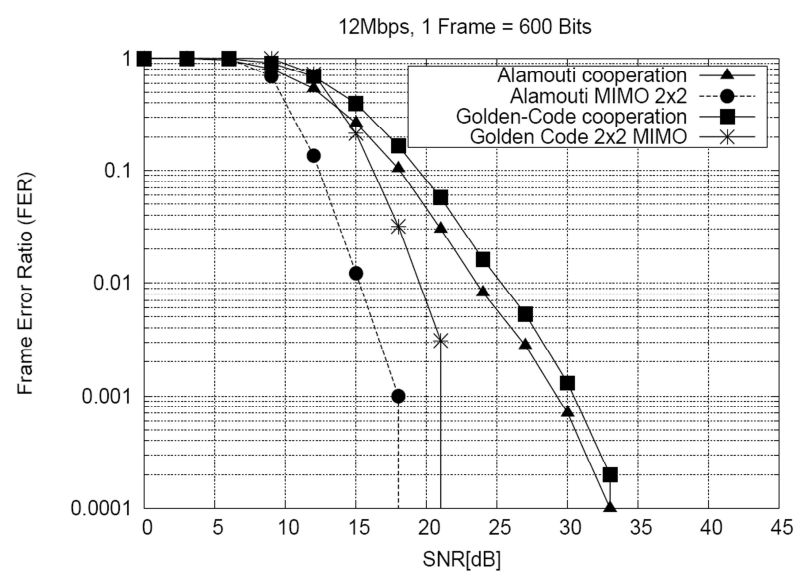

Figure 11. FER versus SNR for STBC in cooperation and MIMO schemes (12Mbps). 


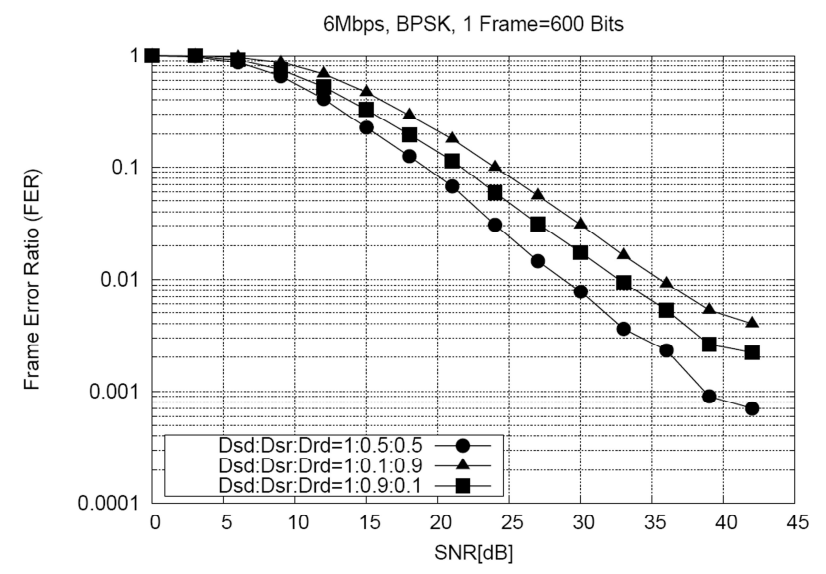

Figure 12. Benefit results when the relay is located between the source and the destination (6Mbps).

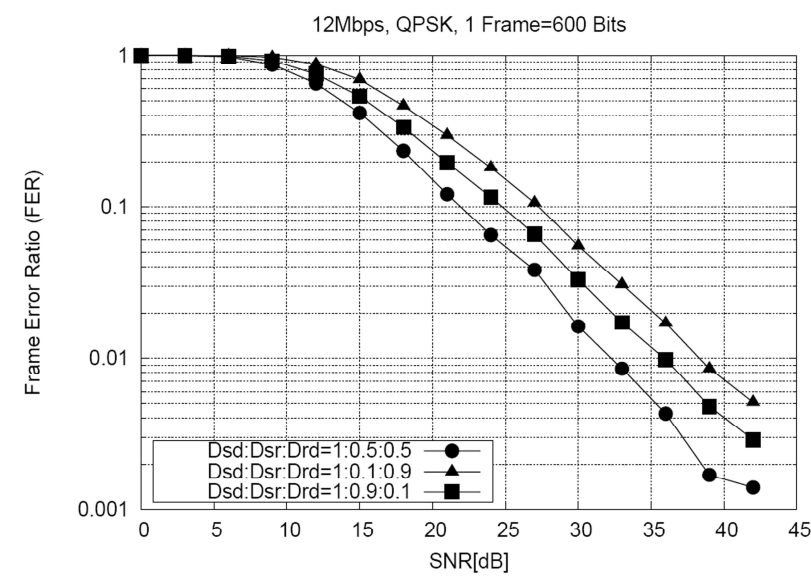

Figure 13. Benefit results when the relay is located between the source and the destination (12Mbps).

The best performance is achieved when the relay is situated in the middle of the source and destination, which means the better channel quality at $S \rightarrow R$ and $R \rightarrow$ $D$. And this can be a rule for a relay-selection method at MAC-layer using the information of PHY-layer.

\section{Conclusions}

This paper describes STBC cooperation in wireless communication, a technique that allows single-antenna mobiles to share their antennas for obtaining some benefits of multiple-antenna systems. The diversity is realized by using a third station as a relay and the STBC methods for information coding. We analyze the performance of two different types of STBC cooperative methods (Alamouti coding and Golden- Code) through the theoretical study and simulations, there is the tradeoff between throughput and reliability during the transmission. The results show that using the STBC cooperative diversity can always increase the performance. Through the analysis of the two methods with the corresponding MIMO systems, we know that the performance of MIMOs is always better than that of cooperation with allowing multiplexing gain. The location of the relay is crucial to the performance.

The best performance was achieved when the relay is in the middle of source and destination. And in general the relay should not be to far from the line between the two terminals.

We believe several areas of future research on cooperative communication will be fruitful. Firstly, the generalization of the one hop space-time coded cooperation to multi-hop case. Most of the research work about cooperative communication concerns the singlehop (single-relay or multi-relay) transmission. Nowadays, multi-hop ad-hoc network can be found in everywhere, and the protocol adapted to multi-hop environment always derives from that of the single-hop. Secondly, the integration and interaction with higher layer network protocols can be explored. Recently, the need for protocol adaptation and code cooperation of wireless communication system suggested a new concept of protocol architecture, named cross-layering architecture. Different protocols implemented at different protocol layers may be designed to have mutually cooperative reactions, based on sharing the information between the different layers. Obviously, a cross-layer approach that is based on metrics computed at physical layer as SNR and minimal distance in the decoding process is under investigation. Such approach will be of a certain interest for MAC and Network levels, taking advantage of the information measured or estimated at the physical layer. Our contribution will concern, mainly, link adaptation and frames scheduling at MAC level. Lastly, generalization of the STBC approach to meshed network while considering multi-channel cooperation, radio resources management and link adaptation will be our crucial objective in perspective.

\section{Acknowledgement}

This work comes within the framework of a project supported by the Agence Nationale de la Recherchel R'eseau National de Recherche en T'el'ecommunications under name RNRT/RADIC-SF/COMSIS and reference ANR-05-RNRT- 014-01.

\section{References}

[1] A. Nosratinia, T. Hunter, and A. Hedayat, "Cooperative communication in wireless networks," IEEE Communications Magazine, Vol. 42, No. 10, pp. 68-73, October 2004.

[2] J. Laneman, G. Wornell, and D. Tse, "An efficient protocol for realizing cooperative diversity in wireless networks," in Proceedings IEEE ISIT, Washington, DC, pp. 294, June 2001. 
[3] J. Laneman, D. Tse, and G. Wornell, "Cooperative diversity in wireless networks: efficient protocols and outage behavior," IEEE Transactions on Information Theory, Vol. 50, No. 12, pp. 3062-3080, December 2004.

[4] J. Laneman and G. Wornell, "Distributed space-time coded protocols for exploiting cooperative diversity in wireless networks," IEEE Transactions on Information Theory, Vol. 49, No. 10, pp. 2415-2425, October 2003.

[5] E. van der Meulen, "Three-terminal communication channels," Advanced Applications Probability, Vol. 3, pp. 120-154, 1971.

[6] G. Kramer and A. van Wijngaarden, "On the white Gaussian multipleaccess relay channel," in Proceedings IEEE International Symposium Information Theory (ISIT), Sorrento, Italy, p. 40, June 2000.

[7] J. Q. Li, K. Letaief, and Z. G. Cao, "Co-Channel interference cancellation for space-time coded OFDM systems," IEEE Transactions on Wireless Communications, Vol. 2, No. 1, pp. 41-49, January 2003.

[8] R. Nabar and H. Bolcskei, "Space-time signal design for fading relay channels," Proceedings IEEE Globecom, San Francisco, CA, Vol. 4, pp. 1952-1956, December 2003.

[9] R. Nabar, H. Bolcskei, and F. Kneubuhler, "Fading relay channels: performance limits and space-time signal design," IEEE Journal on Selected Areas in Communications, Vol. 22, No. 6, pp. 1099-1109, August 2004.

[10] S. Alamouti, "A simple transmit diversity technique for wireless communications," IEEE Journal on Selected Areas in Communications, Vol. 16, No. 8, pp. 14511458, October 1998.

[11] J. Proakis, "Digital communications," Fourth edition. McGraw-Hill, 2001.

[12] J. Belfiore, G. Rekaya, and E. Viterbo, "The Golden Code: A 2x2 full-rate space-time code with nonvanishing determinants," IEEE Transactions on
Information Theory, Vol. 51, No. 4, pp. 1432-1436, April 2005.

[13] H. Yao and G. Wornell, "Achieving the full MIMO diversity-multiplexing frontier with rotation-based spacetime codes," Proceedings of Allerton Conference on Communication, Control and Computing, October 2003.

[14] L. Zheng and D. Tse, "Diversity and multiplexing: A fundamental tradeoff in multiple-antenna channels," IEEE Transactions on Information Theory, Vol. 49, No. 5, pp. 1073-1096, May 2003.

[15] D. Tse, P. Viswanath, and L. Zheng, "DiversityMultiplexing tradeoff in multiple access channels," IEEE Transactions on Information Theory, Vol. 50, No. 9, pp. 1859-1874, September 2004.

[16] Vahid Tarokh, Nambi Seshadri, and A. Calderbank, "Space-time codes for high data rate wireless communication: Performance analysis and code construction," IEEE Transactions on Information Theory, Vol. 44, No. 2, pp. 744-765, March 1998.

[17] V. Tarokh, H. Jafarkhani, and A. Calderbank, "Spacetime block codes from orthogonal designs," IEEE Transactions on Information Theory, Vol. 45, No. 5, pp. 1456-1467, July 1999.

[18] T. Rappaport, "Wireless communications: priciples and practice," New Jersey: Prentice Hall, 1996.

[19] R. Lin and A. Petropulu, "A new wireless medium access protocol based on cooperation," IEEE Transactions on Signal Processing, December 2005.

[20] Y. Zheng and C. Xiao, "Improved models for the generation of multiple uncorrelated Rayleigh fading waveforms," IEEE Communications Letters, Vol. 6, No. 6, June 2002.

[21] J. Chen and K. Djouani. "Space time coding in amplifyand-forward cooperative channel," In Proceedings IEEE International Conference on Wireless Communications, Networking and Mobile Computing, Shanghai, CN, pp. 267-270, September 2007. 